\title{
Transcriptome analysis reveal the mechanism of susceptible wheat seedlings response to Puccinia striiformis f. sp.
}

\section{Rong Liu}

University of the Chinese Academy of Sciences

\section{Yuwei Chen}

University of the Chinese Academy of Sciences

\section{Min Zhou}

University of the Chinese Academy of Sciences

Jing Lu

University of the Chinese Academy of Sciences

\section{Chihong Zhang}

University of the Chinese Academy of Sciences

\section{Lei Zhang ( $\sim$ L95620@126.com )}

University of the Chinese Academy of Sciences

\section{YuWu (D wuyu@cib.ac.cn)}

Chengdu Institution of Biology, Chinese Academy of Sciences

\section{Research article}

Keywords: Wheat, stripe rust, RNA sequencing, yellow rust, CYR34, transcriptome

Posted Date: March 20th, 2020

DOI: https://doi.org/10.21203/rs.3.rs-18149/v1

License: () (1) This work is licensed under a Creative Commons Attribution 4.0 International License. Read Full License 


\section{Abstract}

Background Wheat (Triticum aestivum L.) is most widely cultivated and a major staple food crops in the world. Stripe rust caused by Puccinia striiformis f. sp. tritici (Pst), which significantly reduce yield and quality of wheat. Although some resistant genes have been successfully used in wheat breeding, large of the regulating networks and the underlying molecular mechanisms of Pst response remains unknown. Therefore, to identify differentially expressed genes (DEGs) and regulate network involved in Pst resistance, we sequenced 15 cDNA libraries constructed from wheat seedlings with CYR34 infection.

Results In this study, a highly susceptible cv. Chuanyu12 (CY12) were used to study the transcriptome profiles after inoculated with Pst physiological race CYR34. A total of 13892, 10195, 12268 and 14044 DEGs were investigated at 24h, 48h, 72h and 7days Pst infection, respectively. Certain key genes and pathways responsible for Pst-CYR34 in CY12 were identified. The results revealed that Pst-CYR34 inhibited the DEGs related to energy metabolism, biosynthesis, carbon fixation, phenylalanine metabolism, and plant hormone signaling pathway after Pst inoculation at 24h, 48h, 72h and 7d. These down-regulated DEGs including light-harvesting chlorophyll protein complex in photosystem I and photosystem Il; cytochrome b6/f/ complex, F-type ATPase and photosynthetic electron transport; ethylene, jasmonic acid (JA) and salicylic acid (SA); lignin and flavonoids biosynthesis in CY12. Quantitative Real-time PCR analysis verified the expression patterns of these DEGs.

Conclusions Our results give insights into the foundation for further exploring the molecular mechanism regulating networks of Pst response and pave the way for durable resistant breeding in bread wheat.

\section{Background}

Wheat (Triticum aestivum L.) is the most widely grown and a major staple food crops in the world (Prasad et al. 2019). Wheat stripe rust caused by Puccinia striiformis f. sp. (Pst), one of the most globally devastating fungal disease (Wellings 2011, Wang et al. 2019), which has become a major threat causing massive yield losses of wheat. The Pst races are capable spreads long-distance through seasonal wind and human-assisted movement to other region or continents (Prasad et al. 2019). In China, stripe rust has been considered as the most important fungal disease to decrease wheat production which threatens all wheat cultivars (Wan et al. 2004, Zeng and Luo 2006, Zhou et al. 2019). For its strong adaptive capacity, wheat Pst was evolved fast to become new virulent race then overcome resistant cultivars within a short time, that wheat cultivars with race-specific resistance to stripe rust can retain resistance only several years (Kang et al. 2015).

Previously deployed resistance genes lose effectiveness and yield losses caused by the emergence of new Pst races (Schwessinger 2017). A new Pst race (V26/CYR34) was virulent to resistance gene Yr26 and spread in several major wheat growing regions in the wheat breeding programs (Liu et al. 2010, Wang et al. 2017). Recently, some study reported that about $76 \%$ of current wheat cultivars and breeding lines are susceptible to CYR34 (Han et al. 2015, Bai et al. 2018). However, wheat cultivars with Yr26 is still distributing frequently in China (Wang et al. 2019). Therefore, it is necessary to breed novel resistant cultivars or understand the defense response of wheat against Puccinia striiformis $\mathrm{f}$. sp. before CYR34 causing more lost in wheat production.

Global gene expression methods can be used to elucidate the molecular mechanisms of wheat-fungal interactions, particularly the use of next-generation sequencing technologies to study important non-model hostspathogen systems such as wheat rust (Cantu et al. 2011). Therefore, the main objective of this study are expected 
to provide a better understanding of wheat susceptible/resistance mechanisms response to stripe rust infections, and thus to enhance the yield of wheat. We investigated the phenotypic and co-regulated mRNAs transcriptomic responses of wheat cv. Chuanyu12 (CY12) that show a change in expression pattern after inoculation with PstCYR34 to identify DEGs and related signal pathways to the fungal response. This is the first study to reveal susceptible mechanisms using a global expression profiling strategy in the same wheat genetic background.

\section{Results}

\section{mRNA transcriptome and analysis of CY12}

To elucidate the molecular basis for the stripe rust response in CY12, comparative transcriptome analysis was conducted through RNA sequencing. Both the 0h, 24h, 48h, 72h and 7d Pst treatments leaves were used to investigate early and later response of wheat to Pst-CYR34. Fifteen CDNA libraries, each for CY12 inoculated with Pst-CYR34 and non-infected control at five time points, were characterized by Illumina HiSeq to detect the transcriptome level of gene expression information. After removing low-quality reads and those containing adapter and ploy-N, about 48.0 million (range 47.67 to 48.40 ) clean reads were obtained in per library, among which more than $96.61 \%$ clean reads per library could be mapped to the wheat reference genome (ftp://ftp.ensemblgenomes.Org/pub/plants/release-

37/fasta/triticum_aestivum/dna/Triticum_aestivum.TGACv1.dna.toplevel.fa.gz). The uniquely mapped in CY12_0h, CY12_24h, CY12_48h, CY12_72h and CY12_7d were (86.92 \pm 0.35$) \%,(97.64 \pm 0.2) \%,(86.70 \pm 0.64) \%$, $(87.14 \pm 0.77) \%$ and $(86.55 \pm 0.81) \%$, respectively (Table 1$)$.

\section{DEGs identification}

The DEGs were identified by comparing the FPKMs values of each gene in CY12 with the criteria of fold change $\geq 2$ and $P<0.05$. Then DEGs involved in Pst-CYR34 infection process were screened. The heat map of sample correlations (sample to sample clustering) showed that gene expression values among 15 samples were reproducible between the three biological replicates and batch effects were controlled (Figure S1). The DEGs of CY12 with Pst-CYR34 infection were found for 13892 DEGs (6835 up-regulated and 7057 down-regulated) at 24hpi, 10195 DEGs (6081 up-regulated and 4114 down-regulated) at 48hpi, 12268 DEGs (6494 up-regulated and 5774 down-regulated) at 72hpi and 14044 DEGs (8352 up-regulated and 5692 down-regulated) at 7dpi (Figure 1, Figure 2B).

To examine the unique and shared DEGs in wheat following with Pst-CYR34 infection, the Venn diagram were constructed. All DEGs at Ohpi, 24hpi, 48hpi, 72hpi and 7dpi were shown in Figure 2 (Supplemental File 1). A total of 1653 specific DEGs were identified sharing in each group (Figure 2A). Among them, 620 shared DEGs were upregulated (Figure 2C) and 575 DEGs were down-regulated (Figure 2D) at all time points in CY12. All 1653

differentially expressed genes were show in heatmap (Figure 3). As shown in the figure 3, compare with Ohpi, there were significant changes in the expression of the DEGs after Pst-CYR34 inoculation. Therefore, we conducted following analysis on these differentially expressed genes.

\section{Verification of RNA-Seq by qRT-PCR}

To evaluate the validity of transcriptome profiles from the RNA sequencing analysis, 12 DEGs were randomly selected for qRT-PCR expression analysis. The correlation of RNA-Seq (FPKM) and qRT-PCR are shown in Figure 4 
(Supplemental File 2). The relative expression levels of the genes from qRT-PCR were consistent with those from the RNA-Seq data $(P<0.001)$, which confirming the reliability of the RNA-Seq data.

\section{Gene ontology (GO) and KEGG analysis}

To identify the major functional terms under the Pst-CYR34 infection, GO enrichment analysis was carried out of DEGs (Figure S2). GO enrichment results showed that Pst-CYR34 response DEGs were mainly enriched in signal transduction, energy metabolism, translation, carbohydrate metabolism and amino acid metabolism in CY12 at 24h, 48h, 72h and 7dpi.

For KEGG analysis, Pst-CYR34 induced DEGs in CY12 was notably down-regulated in photosynthesis, plant hormone signal transduction, carbohydrate metabolism, and amino acid metabolism at $24 \mathrm{~h}, 48 \mathrm{~h}, 72 \mathrm{~h}$ and $7 \mathrm{~d}$ time points(Figure 5A-D). The up-regulated genes in CY12 were significantly enrichment in vitamin B6 metabolism, ribosome and RNA degradation (Figure 5E-H). Those results indicate that energy metabolism (including photosynthesis, carbohydrate metabolism) and plant hormone and are important for disease resistance in wheat. Therefore, the down-regulation of the relevant DEGs may cause the cv. CY12 to be susceptible to Pst.

\section{Energy metabolism involved in wheat response to Pst}

The Pst induced DEGs involved in energy metabolism were examined in this study, such as photosynthesis and carbon fixation. Figure 6 (Supplemental File 3) shows the pathway of photosynthesis, carbon fixation and heatmap of DEGs enrichment in those pathways at 0hpi, 24hpi, 48hpi, 72hpi and 7dpi in CY12. Compared with non-infection control, all enrichment DEGs were down-regulated in photosynthesis (KO00195, KO00196) and carbon fixation photosynthetic organisms (K000710) after Pst inoculation (Figure 6C, D, E). Including DEGs of cytochrome b6/f/ complex, F-type ATPase, photosynthetic electron transport, light-harvesting chlorophyll protein complex (LHC) in photosystem $\otimes$ and photosystem $\otimes$ (Figure $6 \mathrm{~A}, \mathrm{~B}$ ). Therefore, we suggest that the photosynthesis related pathway and the Pst-induced expression pattern changes of those DEGs were play an important roles in defense response of Pst-CYR34 infection.

\section{DEGs in plant hormone signaling pathway}

KEGG enrichment analysis exhibited the Pst response of plant hormone signaling interaction in CY12. Compared with control, the DEGs enrichment in plant hormone signaling transduction (KO 04075) were almost downregulated in CY12 after Pst infection. The down-regulated DEGs were mainly in cysteine and methionine metabolism, a-Linolenic acid metabolism and phenylalanine metabolism, which were related to ethylene (ET), jasmonic acid (JA) and salicylic acid (SA) signaling pathway. Then the heatmap and detail flow of DEGs in plant hormone signaling pathway were showed in Figure 7 (Supplemental File 4). Ethylene (ET), jasmonic acid (JA) and salicylic acid (SA) were involved in Pst resistant of wheat. The down-regulated genes eventually involved to regulate disease resistance, senescence and stress response of CY12. This result indicate that these plant hormone were the key factor of plants to responses defense after post inoculation. Otherwise, the plant will lose resistance and the pathogen will successfully invading.

\section{DEGs involved in phenylalanine metabolism}

In present study, we found that phenylalanine pathway was also important for wheat to Pst response. Those DEGs also down-regulated in CY12 at 24hpi, 48hpi, 72hpi and 7dpi (Figure 8A, Supplemental File 5). Although 
there were some cases of up-regulation or non-significant down-regulation for some DEGs at different time points, most of the genes in this pathway were down-regulated in CY12 within Pst infection compared with the noninoculated control.

The enrichment and trend flow of DEGs in phenylalanine metabolism pathway were shown in Figure 8B. Phenylpropanoid firstly biosynthesis phenylalanine, and then with different steps of the pathway catalyzed by four types of enzymes: ammonia lyase (PAL), hydroxycinnamoyl transferase (HCT), cinnamyl alcohol dehydrogenase (CAD) and E1.11.1.7. Finally, the pathway produces three types of monolignols polymerize to form lignin, such as guaiacyl $(\mathrm{G})$, syringyl $(\mathrm{S})$, and p-hydroxyphenyl $(\mathrm{H})$ lignin. While another branch of this pathway was to synthesis of flavonoids and anthocyanins catalyzed by cinnamate 4-hydroxylase (C4H), 4-coumarate-CoA ligase (4CL), chalcone isomerase $(\mathrm{CHI})$ and Chalcone synthase $(\mathrm{CHS})$. This results shown that Pst-CYR34 inoculation decreased lignin and flavonoids biosynthesis in CY12. Therefore, the study indicating that lignin and flavonoids were play an important roles to response defenses and enhancing the resistance of wheat.

\section{Discussion}

In order to meet the future demands of food security, the challenge to wheat production is compounded by effectively controlled wide-spread occurrence of plant diseases (Sharma et al. 2018). In wheat, the most destructive diseases is stripe rust that can cause severe yield losses when susceptible cultivars are grown in the field (Wan et al. 2004). However, the information of plant defense regulation network is still limited, such as activation and down-regulation of Pst induced genes to fine-tune the plant defense response remains unclear. It is well known that cultivars with race-specific resistance usually become susceptible within a few years due to the rapid evolution of virulent races of Pst (Line et al. 1995). Therefore, it is essential to understand the wheat susceptible mechanisms to developing new strategies for improving disease resistance in wheat.

Plants have a complex regulatory networks to regulate growth and to defense response of various biotic and abiotic stresses. The plant hormone, like gibberellins, auxins, cytokinins, jasmonic acid (JA), ethylene (ET), brassinosteroids (BRs), salicylic acid (SA), abscisic acid (ABA), and strigolactone are play essential roles in whole plant's life (Chow et al. 2006). During defense, phytohormone-mediated signaling is critically important for increasing resistance to plant (Hussain et al. 2018, Verma et al. 2016). Such as, JA, SA, ET, ABA, and gibberellins can provide broad biochemical resistance and reduce pathogen growth in plant (Robert et al. 2011). Jasmonic acid (JA) was proved involving in plant development and certain stress responses, such as attack by herbivores and pathogen infection (Wasternack et al. 2007, Wu et al. 2009, Browse 2009). Phytohormone ethylene (ET) plays role in plant development regulation and stress resistance (Ozimek et al. 2018). In our results, Ethylene and jasmonic acid were involved in Pst response of wheat. And all of DEGs were down-regulated in those pathways, which eventually involved to regulate senescence and stress response of wheat to defense the CYR34 infection.

The small plant signaling hormone molecule salicylic acid (SA) is essential in plant disease resistance (Wang et al. 2010), which required for both local defense and systemic acquired resistance (Vlot et al. 2009). Plants accumulate SA by microbial pathogens infections (Loake et al. 2007, Vlot et al. 2008). Higher level of SA associate with higher disease resistance in plant. Otherwise, the plants fail to accumulate SA are more susceptible to virulent pathogens (Dempsey et al. 1999). Certain research reported that spot blotch-resistant wheat specifically elicit the change in the SA levels after pathogen infection, which reprograms the expression of several defense associated genes, ultimately conferring resistance (Sahu et al. 2016). However, susceptible wheat failed to display 
a similar response, indicating that spot blotch induced SA accumulation is an important event to regulate spot blotch resistance in wheat. Similar with previous study, we also found that the DEGs were all down-regulated in SA signal pathway with Pst-CYR34 infection in susceptible wheat cv. CY12. Pathogen induced SA involved to produce anti-microbial pathogenesis-related proteins, thus to promote plant immunity against microbial pathogens through combined with anti-microbial proteins (Wang et al. 2005). According to the result of RNA_seq, we suggest that the mechanism of wheat susceptible to CYR34 may cause by ET, JA and SA pathway suppression. As a signaling molecule, they may confers resistance in wheat.

Photosynthesis and carbon metabolism dominate plant energy metabolism, and more than $90 \%$ of grain yield is produced by photosynthesis (Makino et al. 2011). Photosynthetic activities are affected by many abiotic and biotic stresses. However, so far, most studies on wheat photosynthesis under environment stresses focused on abiotic stresses (Yang et al. 2008). In contrast, only a few studies have been conducted on the relationship between the photosynthesis and biotic stresses. Even though a previous study indicated that pathogen infection may result in changes in photosynthesis (Wang et al. 2000), the detailed effects of wheat stripe rust on photosystem I (PS I) and photosystem II (PS II) are poorly understood. To better understand wheat stripe rust response mechanism, we investigated the DEGs enrichment in photosynthetic and carbon fixation pathway in susceptible wheat cultivars that were infected by new stripe rust races CYR34. A previous study revealed that Pst infection may result in a decrease in Photosynthesis related protein (D1) in susceptible wheat (Shen et al. 2008). This finding is consistent with our results. In the current study, we found that the DEGs of Photosynthesis and carbon fixation pathway were all down-regulated in the susceptible wheat (CY12) after inoculated with Pst-CYR34, the DEGs including cytochrome b6/f/ complex, F-type ATPase, photosynthetic electron transport, photosystem $\otimes$

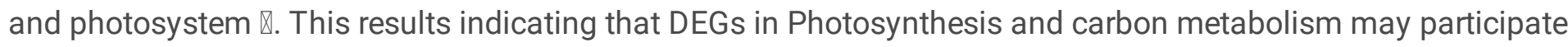
in the regulation of wheat resistance to stripe rust.

Phenylpropanoid metabolism is the most important secondary metabolic pathway involved in plant defense against biotic and abiotic stresses (La et al. 2004). The main branches of the phenylpropanoid pathway were led to the synthesis of lignin and flavonoid. Certain researches provided evidence to suggest that lignin, flavonoid and phenolic compounds play important roles during the plant defense response to pathogen, they accumulate in plant can enhance plants resistance (Harborne 1999). However, rarely studies on the overall analysis of transcriptome changes of those genes in wheat. In this study, we found that phenylalanine pathway DEGs were down-regulated in CY12 with Pst infection. Which led to lignin and flavonoids biosynthesis decreased in CY12. Lignin can provides mechanical strength and reinforces cell walls to provide a physical barrier to limit pathogen infection (Bonello and Blodgett 2003). And also preventing the transfer of water and nutrients from plant to pathogen, to restrict pathogen growth (Hu et al. 2018). Therefore, the plant will lose resistance and the pathogen will successfully invading by decreased lignin and flavonoids biosynthesis.

\section{Methods}

\section{Plant materials and fungal treatment}

The wheat cv. Chuanyu12 (CY12) was used in this study, which obtained from Chengdu Biology of Sciences, Chinese Academy of Sciences. CY12 is developed by our research group and highly susceptible to currently predominant stripe rust, such as new Pst-race CYR34 (Liu et al. 2020). Artificial inoculation at seedling stage was conducted under a controlled greenhouse condition in Gansu Academy of Agricultural Sciences (GAAS). Two- 
week old seedlings were inoculated with Pst-CYR34. The wheat cv. Mingxian169 was used to monitor inoculation efficiency. The wheat leaves of $\mathrm{CY} 12$ were collected at $0 \mathrm{~h}, 24 \mathrm{~h}, 48 \mathrm{~h}, 72 \mathrm{~h}$ and 7 days post-inoculation (each time point with 3 biological replicates). All leaf samples were frozen in liquid nitrogen and stored in a $-80^{\circ} \mathrm{C}$ refrigerator for transcriptome sequencing.

\section{RNA Isolation and cDNA library construction for RNA Sequencing}

Total RNA of wheat leaves was extracted using the mirVana miRNA Isolation Kit (Ambion) following the manufacturer's protocol. RNA integrity was evaluated using the Agilent 2100 Bioanalyzer (Agilent Technologies, Santa Clara, CA, USA). The samples with RNA Integrity Number (RIN) $\geq 7$ were subjected to the subsequent analysis. Total RNA from the wheat leaves was to break into short fragments at a suitable temperature in the thermomixer. Then purified fragment mRNA was used to synthesize first strand cDNA and second strand cDNA. The libraries were constructed using TruSeq Stranded mRNA LTSample Prep Kit (Illumina, San Diego, CA, USA) according to the manufacturer's instructions. Then $15 \mathrm{cDNA}$ libraries were sequenced on the Illumina sequencing platform (HiSeqTM 2500 or Illumina HiSeq X Ten) and 125bp/150bp paired-end reads were generated.

The transcriptome sequencing were conducted by OE biotech (Shanghai, China). Raw data (raw reads) were processed using Trimmomatic (Bolger et al. 2014). The reads containing ploy- $\mathrm{N}$ and the low quality reads were removed to obtain the clean reads. Then, all clean reads used to subsequent analyses and mapped to reference genome using hisat2 (Kim et al. 2015).

\section{Differential expression genes (DEGs), Gene Ontology (GO) and KEGG Analysis}

FPKM (Roberts et al. 2011) value of each gene was calculated using cufflinks (Trapnell et al. 2010), and the read counts of each gene were obtained by htseq-count (Anders et al. 2015). Differentially expressed genes (DEGs) were analysis and identified using the DEGseq R package. DEGs were selected with a $|\log 2 \mathrm{FC}|>1$ and $\mathrm{Q}<0.005$ as the thresholds, which tested whether each gene was affected by genotype and time point. Hierarchical cluster analysis of DEGs was performed to explore genes expression pattern. And $P$ values were adjusted using the Benjamini-Hochberg correction for multiple testing (Benjamini and Hochberg 1995). GO enrichment and KEGG (Kanehisa et al. 2008) pathway enrichment analysis of DEGs were respectively performed using $\mathrm{R}$ based on the hypergeometric distribution during CYR34 infection.

\section{qRT-PCR analysis}

Twelve DEGs were randomly selected for qRT-PCR analysis, and GAPDH was used as the internal reference gene. The primers were designed based on the sequence of genes using Primer 5.0 and are listed in Table S1. The RNA extracted from $\mathrm{CY} 12$ at $0 \mathrm{~h}, 24 \mathrm{~h}, 48 \mathrm{~h}, 72 \mathrm{~h}$ and 7days were used to synthesize first-strand cDNA with HiScriptll Q RT SuperMix (Vazyme, R223-1) following the manufacturer's instructions. The qRT-PCR was performing with SYBR® Green PCR kit (Qiagen, 204054) according to the manufacturer's instructions. The experimental conditions were set as follows: 45 cycles at $95{ }^{\circ} \mathrm{C}$ for $20 \mathrm{~s}, 55^{\circ} \mathrm{C}$ for $20 \mathrm{~s}$, and $72{ }^{\circ} \mathrm{C}$ for $20 \mathrm{~s}$. All genes were repeated 3 times in this study. The mRNA expression level of the genes were calculated with the $2^{-\Delta \Delta c t}$ method (Schmittgen and Livak 2008). Each wheat leaf sample was repeated 3 times. The correlation between RNA-seq and qRT-PCR results was analyzed using these values R package version 3.1.3 (http://cran.r-project.org/). The normalized values of relative expression and RPKM values were calculated using log2 (fold change) measurements.

\section{Statistical analysis of data}


Data of three biological repeats were analyzed using GraphPad Prism 5, Excel 2013 and SPSS 20.0 and rendered as means \pm SD. One-way ANOVA followed by Tukey's significant difference test at $p<0.05$. All data had 3 biological repeats. Differentially expressed genes were defined as genes with FDR $<0.001$ and fold change $>2$-fold. A p-value $<0.05$ was considered significant when identifying enriched GO terms, and a p-value $<0.05$ was considered indicative of significantly enriched KEGG pathways.

\section{Additional Files}

Table S1. List of primers used for the relative quantification of gene transcripts (XLSX 10 kb). Figure S1. Hierarchical clustering analysis of differentially expressed genes (DEGs) in CY12 at 24h (A), 48h (B), 72h (C) and 7d (D) under Pst-CYR34 infection, respectively. (DOCX $626 \mathrm{~kb}$ ). Supplementary File1: The data of all DEGs at Ohpi, 24hpi, 48hpi, 72hpi and 7dpi in CY12 (XLSX 467 kb). Supplementary File2: The DEGs GO used for GO enrichment analysis (XLSX $14 \mathrm{~kb}$ ). Supplementary File3: The DEGs enrichment in photosynthesis, carbon fixation pathway (XLSX 16 kb). Supplementary File4: The DEGs in plant hormone signaling pathway (XLSX 17 kb). Supplementary File5: The DEGs enrichment in phenylalanine pathway (XLSX 20 kb).

\section{Abbreviations}

Yr. Yellow rust; Pst. Puccinia striiformis f. sp. tritici; CY12: Chuanyu12; DEGs: different expression genes; PAL: ammonia lyase; HCT: hydroxycinnamoyl transferase; CAD: cinnamyl alcohol dehydrogenase; G: guaiacyl; S: syringyl; H: p-hydroxyphenyl, JA: jasmonic acid, ET: ethylene, BRs: brassinosteroids, SA: salicylic acid, ABA abscisic acid, $\mathrm{C} 4 \mathrm{H}$ : cinnamate 4-hydroxylase, 4CL: 4-coumarate-CoA ligase, $\mathrm{CHI}$ : chalcone isomerase, $\mathrm{CHS}$ : chalcone synthase.

\section{Declarations}

\section{Ethics approval and consent to participate}

Not applicable.

\section{Consent for publication}

Not applicable.

\section{Availability of data and materials}

The dataset and materials presented in the investigation is available by request from the corresponding author.

\section{Competing interests}

The authors declare that they have no competing interests. There was no competing Interests in this work.

\section{Funding}

This work was supported by the "13th Five-year Plan" for National Key Research and Development (Grant numbers 2017YFD0100902). Innovation Academy for Seed Design, CAS. The "13th Five-year Plan" for Wheat 
Crops Breeding in Sichuan Province. The funders had no role in the study design, data collection, and analysis, decision to publish or preparation of the manuscript.

\section{Authors' Contributions}

YW and LZ provided experimental design and plant material. RL performed experiments and RNA_Seq data analyses. $R L$ and $J L$ were identification phenotype of wheat and data analysis. $R L$ wrote the manuscript. $M Z, C H Z$ and YFL were read this article and modified. All authors read and approved the final manuscript.

\section{Acknowledgements}

Thanks Cao Shiqin, Jia Qiuzhen and Wang Xiaoming of Gansu Academy of Agricultural Sciences for helping inoculate stripe rust races (Pst-CYR34) and identification during seedling stage, and thank Xia Xianquan (Sichuan Academy of Agricultural Sciences) for helping to conduct field inoculation.

\section{References}

Anders S, Pyl PT, Huber W (2015) HTSeq-a Python framework to work with high-throughput sequencing data. Bioinformatics 31(2): 166-169.

Bai BB, Liu TG, Liu B, Gao L, Chen WQ (2018) High relative parasitic fitness of G22 derivatives is associated with the epidemic potential of wheat stripe rust in China. Plant Disease 102(3): 483-487.

Benjamini Y and Hochberg Y (1995) Controlling the false discovery rate-a practical and powerful approach to multiple testing. Journal of the Royal Statistical Society Series B-Statistical Methodology 57(1): 289-300.

Bolger AM, Lohse M, Usadel B (2014) Trimmomatic: a flexible trimmer for Illumina sequence data. Bioinformatics 30(15): 2114-2120.

Bonello P, Blodgett JT (2003) Pinus nigra-Sphaeropsis sapinea as a model pathosystem to investigate local and systemic effects of fungal infection of pines. Physiol Mol Plant Pathol 63: 249-261.

Browse J (2009) Jasmonate passes muster: a receptor and targets for the defence hormone. Annual Review of Plant Biology 60:183-205.

Cantu D, Govindarajulu M, Kozik A, Wang M, Chen X, Kojima KK, Jurka J, Michelmore RW, Dubcovsky J (2011) Next generation sequencing provides rapid access to the ggenome of Puccinia striiformis $f$. sp tritici, the causal agent of wheat stripe rust. Plos One 6(8).

Chow B, McCourt P (2006) Plant hormone receptors: perception is everything. Genes and Development 20:19982008.

Dempsey DMA, Shah J, Klessig DF (1999) Salicylic acid and disease resistance in plants. CRC. Crit. Rev. Plant Sci. $18,547-575$.

Han DJ, Wang QL, Chen XM, Zeng QD, Wu JH, Xue WB, Zhan GM, Huang LL, Kang ZS (2015) Emerging Yr26virulent races of Puccinia striiformis $\mathrm{f}$. tritici are threatening wheat production in the Sichuan basin, China. Plant Disease 99(6): 754-760. 
Harborne JB (1999) The comparative biochemistry of phytoalexin induction in plants. Biochemical Systematics \& Ecology 27(4): 335-367.

Hu Q, Min L, Yang XY, Jin SX, Zhang L, Li YY, Ma YZ, Qi XW, Li DQ, Liu HB, Lindsey K, Zhu LF, Zhang XL (2018). Laccase GhLac1 modulates broad-spectrum biotic stress tolerance via manipulating phenylpropanoid pathway and jasmonic acid synthesis. Plant Physiology 176 (2): 1808-1823.

Hussain A, Li X, Weng Y, Liu Z, Ashraf MF, Noman A, Yang S, Ifnan M, Qiu S, Yang Y (2018) CaWRKY22 acts as a positive regulator in pepper response to Ralstonia solanacearum by constituting networks with CaWRKY6, CaWRKY27, CaWRKY40, and CaWRKY58. Int. J. Mol. Sci 19:1426.

Kanehisa M, Araki M, Goto S, Hattori M, Hirakawa M, Itoh M, Katayama T, Kawashima S, Okuda S, Tokimatsu T, Yamanishi Y (2008) KEGG for linking genomes to life and the environment. Nucleic Acids Research 36: D480D484.

Kang Z, Wang X, Zhao J, Tang C, Huang L (2015) Advances in research of pathogenicity and virulence variation of the wheat stripe rust fungus Puccinia striiformis f. sp. tritici. Scientia Agricultura Sinica 48(17): 3439-3453.

Kim D, Langmead B, Salzberg SL (2015) HISAT: a fast spliced aligner with low memory requirements. Nature Methods 12(4): 357-U121.

La Camera S, Gouzerh G, Dhondt S, Hoffmann L, Fritig B, Legrand M, Heitz T (2004) Metabolic reprogramming in plant innate immunity: the contributions of phenylpropanoid and oxylipin pathways. Immunol Rev 198: 267-284

Line RF, Chen XM (1995) Successes in breeding for and managing durable resistance to wheat rusts. Plant Dis. 79 1254-1255.

Liu TG, Peng YL, Chen WQ, Zhang ZY (2010) First detection of virulence in Puccinia striiformis f. sp tritici in China to resistance genes Yr24 (=Yr26) present in wheat cultivar Chuanmai 42. Plant Disease 94(9): 1163-1163.

Liu R, Lu J, Zhou M, Zheng SG, Liu ZH, Zhang CH, Du M, Wang MX, Li YF, Wu Y, Zhang L (2020) Developing stripe rust resistant wheat (Triticum aestivum $\mathrm{L}$.) lines with gene pyramiding strategy and marker-assisted selection. Genetic Resources and Crop Evolution 67: 381-391.

Loake G, Grant M (2007) Salicylic acid in plant defence-the players and protagonists. Curr. Opin. Plant Biol10:466-472.

Makino A (2011) Photosynthesis, grain yield, and nitrogen utilization in rice and wheat. Plant Physiol 155:125129.

Ozimek E, Jaroszuk-Ściseł J, Bohacz J, Korniłłowicz-Kowalska T, Tyśkiewicz R, Słomka A, Nowak A, Hanaka A (2018) Synthesis of Indoleacetic Acid, Gibberellic Acid and ACC-Deaminase by Mortierella Strains Promote Winter Wheat Seedlings Growth under Different Conditions. Int J Mol Sci 19(10): 3218.

Prasad P, Savadi S, Bhardwaj SC, Gangwar OP, Kumar S (2019) Rust pathogen effectors: perspectives in resistance breeding. Planta 250(1): 1-22. 
Roberts A, Trapnell C, Donaghey J, Rinn JL, Pachter L (2011) Improving RNA-Seq expression estimates by correcting for fragment bias. Genome Biology 12(3).

Robert-Seilaniantz A, Grant M, Jones JD (2011) Hormone crosstalk in plant disease and defense: more than just jasmonate-salicylate antagonism. Annual Review of Phytopathology 49, 317-343.

Sahu R, Sharaff M, Pradhan M, Sethi A, Bandyopadhyay T, Mishra VK (2016) Elucidation of defense-related signaling responses to spot blotch infection in bread wheat (Triticum aestivum L.). Plant J 86, 35-49.

Santner A, Calderon-Villalobos LI, Estelle M (2009) Plant hormones are versatile chemical regulators of plant growth. Nature Chemical Biology 5:301-307.

Schmittgen TD, Livak KJ (2008) Analyzing real-time PCR data by the comparative C-T method. Nature Protocols 3(6): 1101-1108.

Schwessinger B (2017) Fundamental wheat stripe rust research in the 21 st century. New Phytologist 213(4): 1625-1631.

Sharma S, Sahu R, Navathe S, Mishra V, Chand R, Singh P, Joshi A, Pandey S (2018) Natural variation in elicitation of defense-signaling associates to field resistance against the spot blotch disease in bread wheat (Triticum aestivum L.). Front Plant Sci 9: 636.

Shen X, Li HY, Jia QZ, Hong HQ, Li MQ, Liang HG (2008) Influence of wheat (Triticum aestivum L.) stripe rust infection of photosynthetic function and expression protein D1 of wheat leaves. Acta Ecol. Sin 28: 669-676.

Trapnell C, Williams BA, Pertea G, Mortazavi A, Kwan G, J van BarenM, Salzberg SL, Wold BJ, Pachter L (2010) Transcript assembly and quantification by RNA-Seq reveals unannotated transcripts and isoform switching during cell differentiation. Nature Biotechnology 28(5): 511-U174.

Verma V, Ravindran P, Kumar PP (2016) Plant hormone-mediated regulation of stress responses. BMC Plant Biol 16:86.

Vlot AC, Klessig DF, Park SW (2008) Systemic acquired resistance: the elusive signal(s) Curr. Opin. Plant Biol. 11: 436-442.

Vlot AC, Dempsey DMA, Klessig DF (2009) Salicylic acid, a multifaceted hormone to combat disease. Annu. Rev. Phytopathol 47: 177-206.

Wan AM, Zhao ZH, Chen XM, He ZH, Jin SL, Jia QZ, Yao G, Yang JX, Wang BT, Li GB, Bi YQ, Yuan ZY (2004) Wheat stripe rust epidemic and virulence of Puccinia striiformis f. sp tritici in China in 2002. Plant Disease 88(8): 896904.

Wang CZ, Gao F, Wu JG, Dai JL, Wei CH, Li Y (2010) Arabidopsis putative deacetylase AtSRT2 regulates basal defense by suppressing PAD4, EDS5 and SID2 expression. Plant Cell Physiol 51(8): 1291-1299.

Wang CM, Shi DJ, Zhu SF, Tian B, Wei NS (2000) Effects of cucumber mosaic virus infection of photosynthetic activities of tobacco leaves and chloroplasts. Acta Bot. Sin 42: 388-392. 
Wang D, Weaver ND, Kesarwani M, Dong X (2005) Induction of protein secretory pathway is required for systemic acquired resistance. Science 308: 1036-1040.

Wang L, Tang X, Wu J, Shen C, Dai M, Wang Q, Zeng Q, Kang Z, Wu Y, Han D (2019) Stripe rust resistance to a burgeoning Puccinia striiformis f. sp. tritici race CYR34 in current Chinese wheat cultivars for breeding and research. Euphytica 215(4).

Wang Y, Xie J, Zhang H, Guo B, Ning S, Chen Y, Lu P, Wu Q, Li M, Zhang D, Guo G, Zhang Y, Liu D, Zou S, Tang J, Zhao H, Wang X, Li J, Yang W, Cao T, Yin G, Liu Z (2017) Mapping stripe rust resistance gene YrZH22 in Chinese wheat cultivar Zhoumai 22 by bulked segregant RNA-Seq (BSR-Seq) and comparative genomics analyses. Theoretical and Applied Genetics 130 (10): 2191-2201.

Wasternack C (2007) Jasmonates: an update on biosynthesis, signal transduction and action in plant stress response, growth and development. Annals of Botany100:681-697.

Wellings CR (2011) Global status of stripe rust: a review of historical and current threats. Euphytica 179 (1): $129-$ 141.

Wu J, Baldwin IT (2009) Herbivory-induced signalling in plants: perception and action. Plant, Cell and Environment 32:1161-1174.

Yang CW, Wang P, Li CY, Shi DC, Wang DL (2008) Comparison of effects of salt and alkali stresses on the growth and photosynthesis of wheat. Photosynthetica 46: 107-114.

Zeng SM, Luo Y (2006) Long-distance spread and interregional epidemics of wheat stripe rust in China. Plant Disease 90 (8): 980-988.

Zhou X, Hu Li TX, Yu M, Li Y, Yang S, Huang K, Han D, Kang Z (2019) Genome-wide mapping of adult plant stripe rust resistance in wheat cultivar Toni. Theoretical and Applied Genetics 132 (6): 1693-1704.

\section{Tables}

Table 1 Read statistics in 15 RNA sequencing libraries

\begin{tabular}{|c|c|c|c|c|c|c|c|}
\hline Sampe & Raw reads (M) & Clean reads $(\mathrm{M})$ & Q30 (\%) & GC (\%) & Total mapped (\%) & Multiple mapped (\%) & Uniquely mapped (\%) \\
\hline CY12_0h & $49.2 \pm 0.06$ & $47.85 \pm 0.19$ & $94.8 \pm 0.17$ & $54.38 \pm 0.98$ & $97.23 \pm 0.16$ & $10.3 \pm 0.49$ & $86.92 \pm 0.35$ \\
\hline CY12_24h & $49.51 \pm 0.9$ & $47.97 \pm 1.5$ & $94.68 \pm 0.72$ & $53.07 \pm 0.24$ & $96.61 \pm 0.03$ & $9.97 \pm 0.23$ & $97.64 \pm 0.2$ \\
\hline CY12_48h & $49.18 \pm 0.38$ & $47.67 \pm 0.56$ & $94.46 \pm 0.75$ & $54.28 \pm 0.39$ & $96.83 \pm 0.37$ & $10.14 \pm 0.62$ & $86.7 \pm 0.64$ \\
\hline CY12_72h & $49.59 \pm 0.91$ & $48.05 \pm 0.52$ & $94.41 \pm 0.83$ & $53.81 \pm 0.66$ & $96.87 \pm 0.26$ & $9.72 \pm 0.61$ & $87.14 \pm 0.77$ \\
\hline CY12_7d & $49.56 \pm 0.87$ & $48.4 \pm 0.75$ & $94.83 \pm 0.34$ & $54.09 \pm 0.64$ & $97.02 \pm 0.31$ & $10.47 \pm 0.99$ & $86.55 \pm 0.81$ \\
\hline
\end{tabular}

\section{Figures}


A

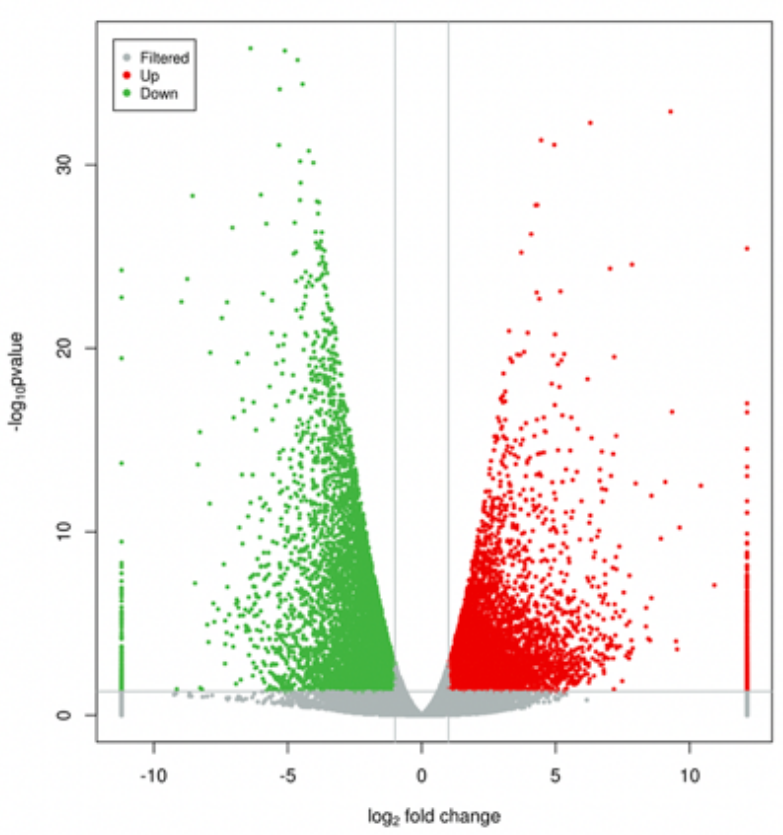

C

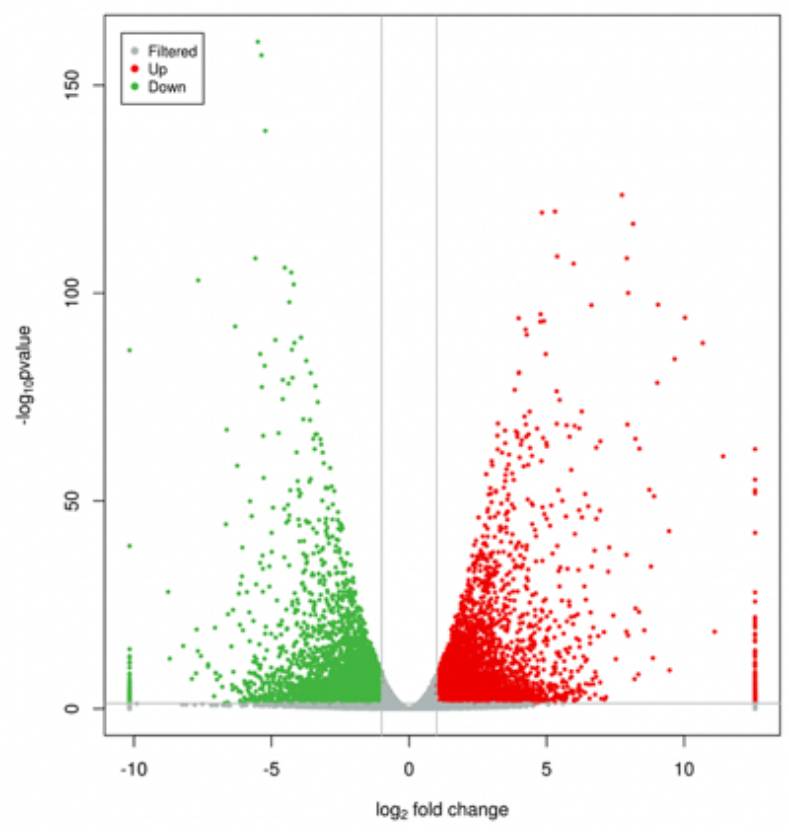

B CY12_c-vs-Cr12_a : pvalue $<0.05 \& \&|\log 2 \mathrm{FC}|>1$

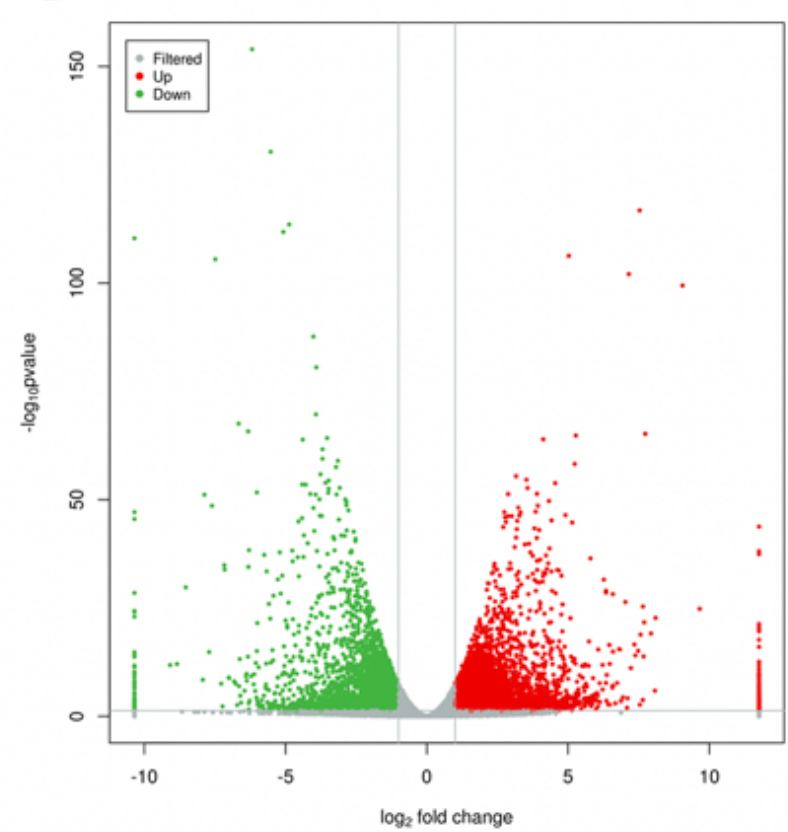

$\mathrm{D}$

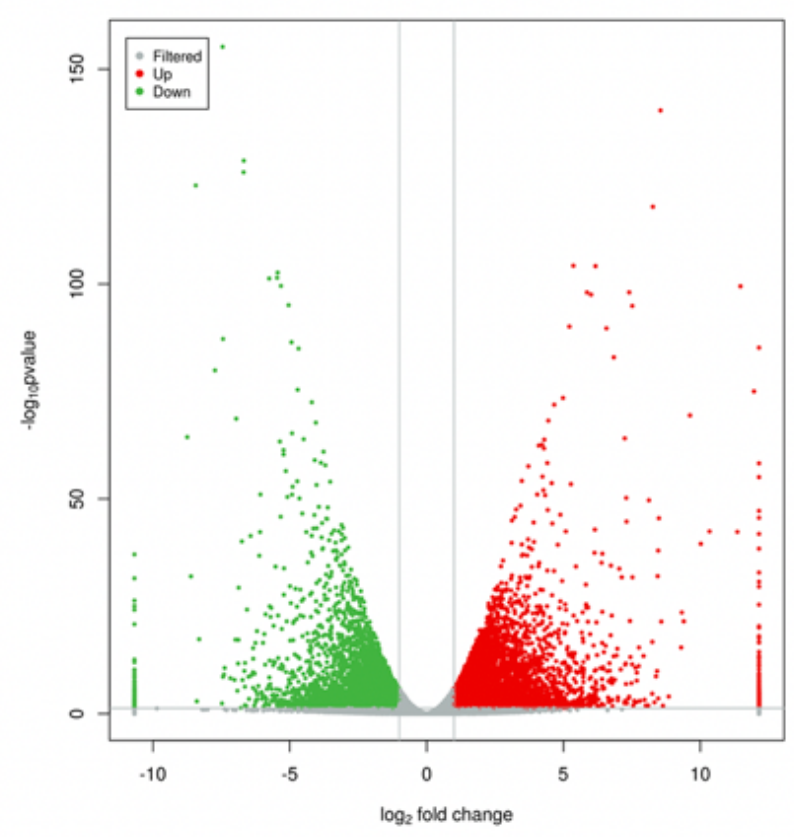

\section{Figure 1}

Volcano plots of differentially expressed genes with Pst-CYR34 infection at 0h, 24h, 48h, 72h and 7d time points in CY12: (A) 24h of Pst-CYR34 infection; (B) 48h of Pst-CYR34 infection; (C) 48h of Pst-CYR34 infection; and (D) 7d of Pst-CYR34 infection. CY12a, CY12b, CY12c, CY12d and CY12e represent different time points of collecting sample, a-0h, b-24h, c-48h, d-72h and d-7days after CYR34 infection. 
A

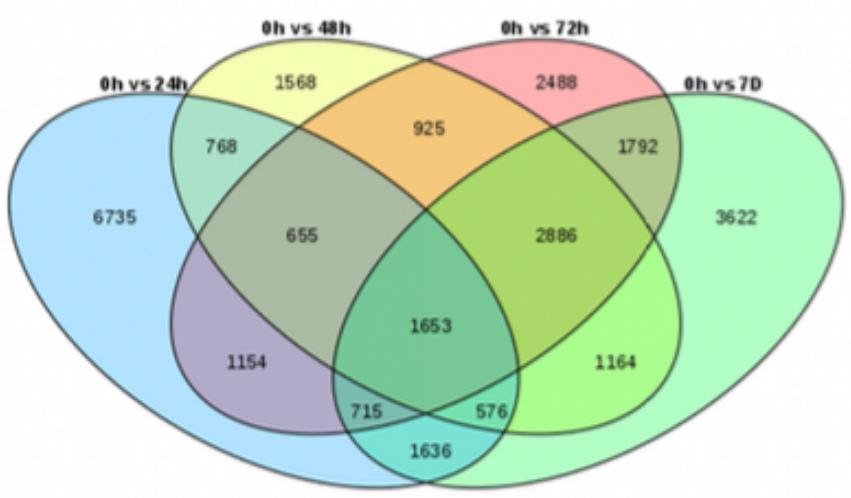

C

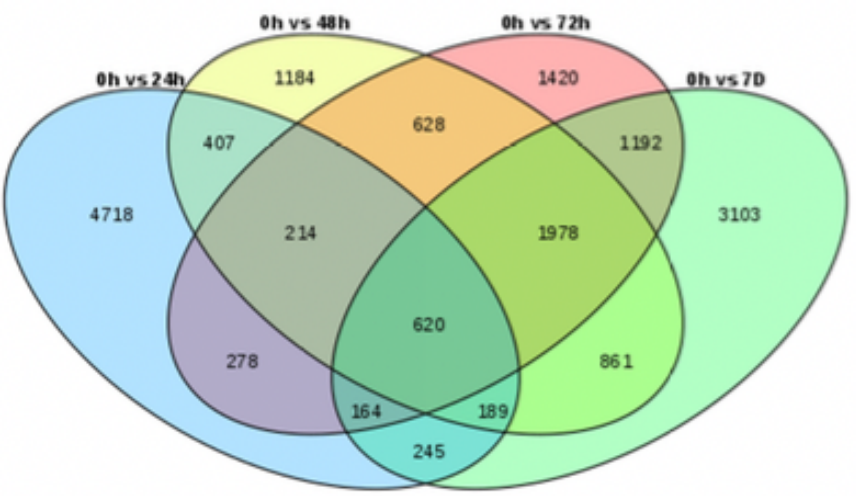

B

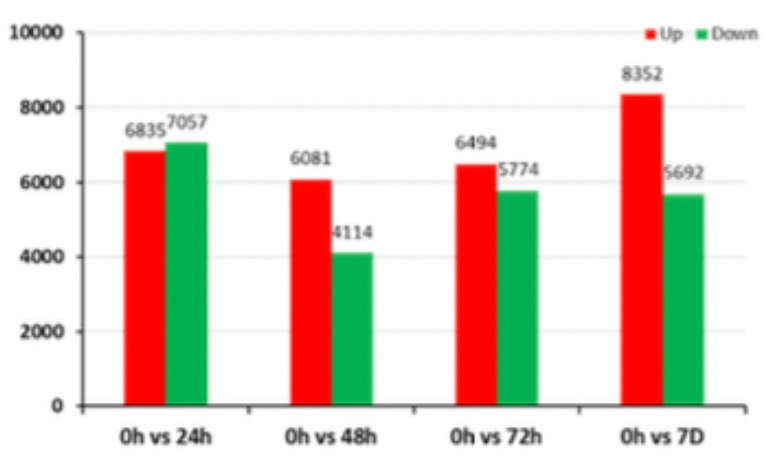

D

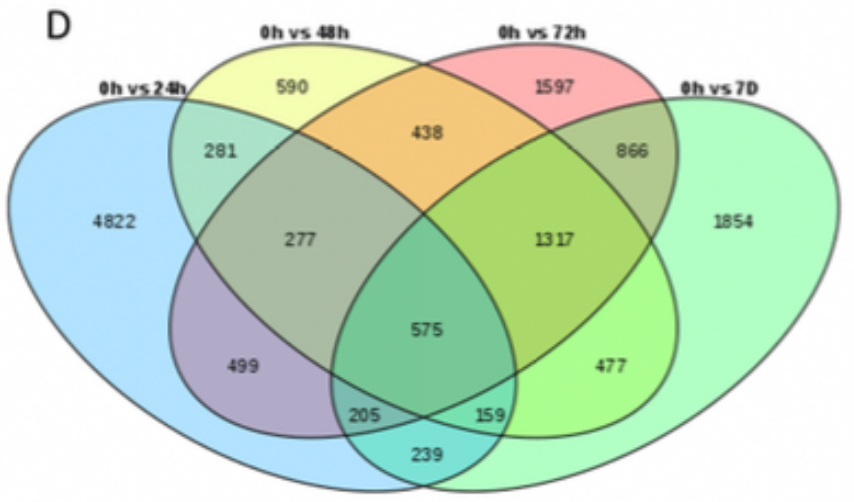

Figure 2

Venn diagrams of differentially expressed genes (DEGs) in CY12. Venn diagram showing the DEGs between PstCYR34 treatment at 0hpi, 24hpi, 48hpi, 72hpi and 7dpi time points in CY12 (A), the number of DEGs up- or downregulated $(B)$ and the Venn diagrams of up-regulated DEGs (C) and down-regulated DEGs (D) at each time points. 


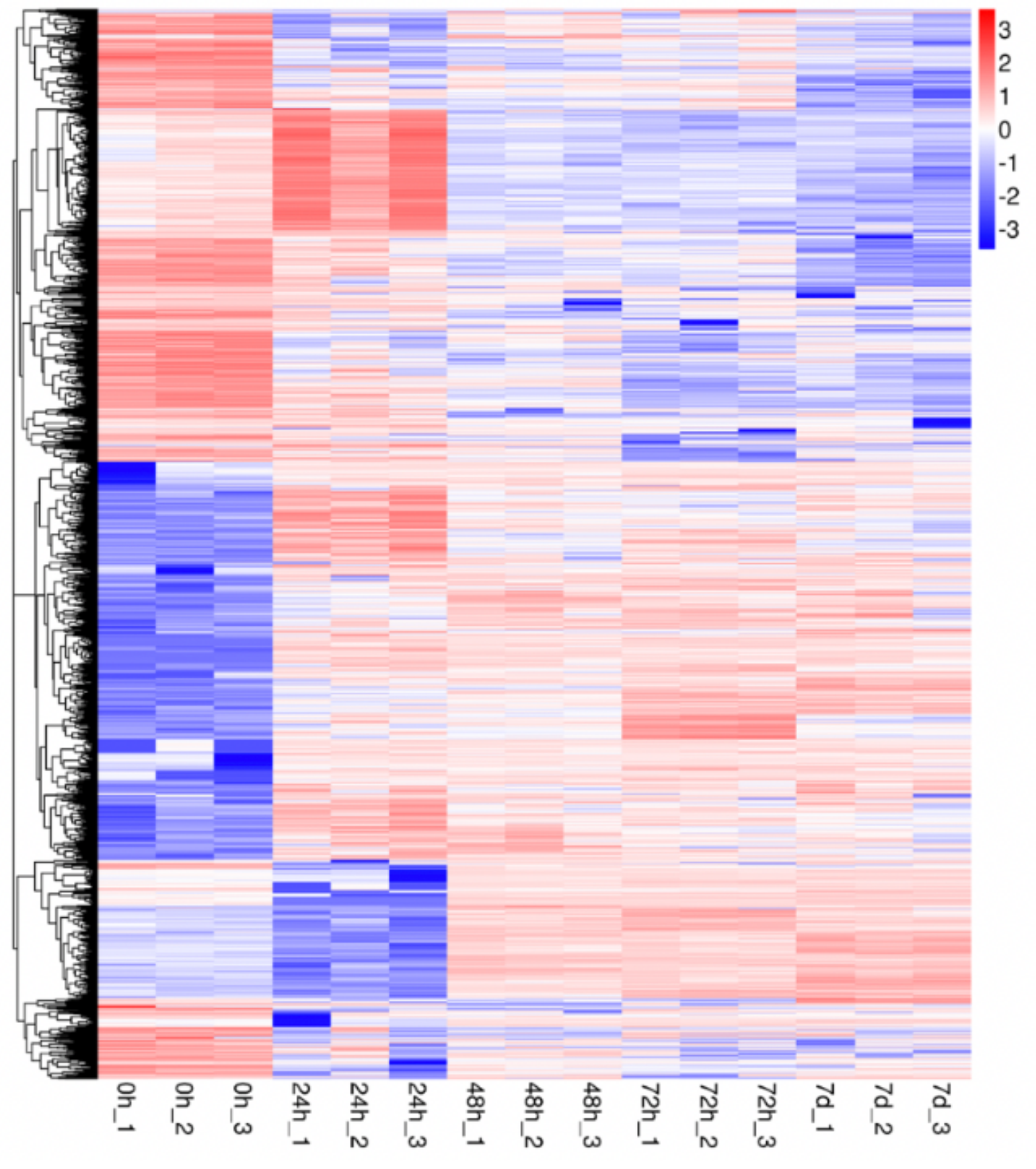

Figure 3

Heatmap of 1653 differentially expressed genes shared in each group at $0 \mathrm{~h}, 24 \mathrm{~h}, 48 \mathrm{~h}, 72 \mathrm{~h}$ and $7 \mathrm{~d}$ time points. 

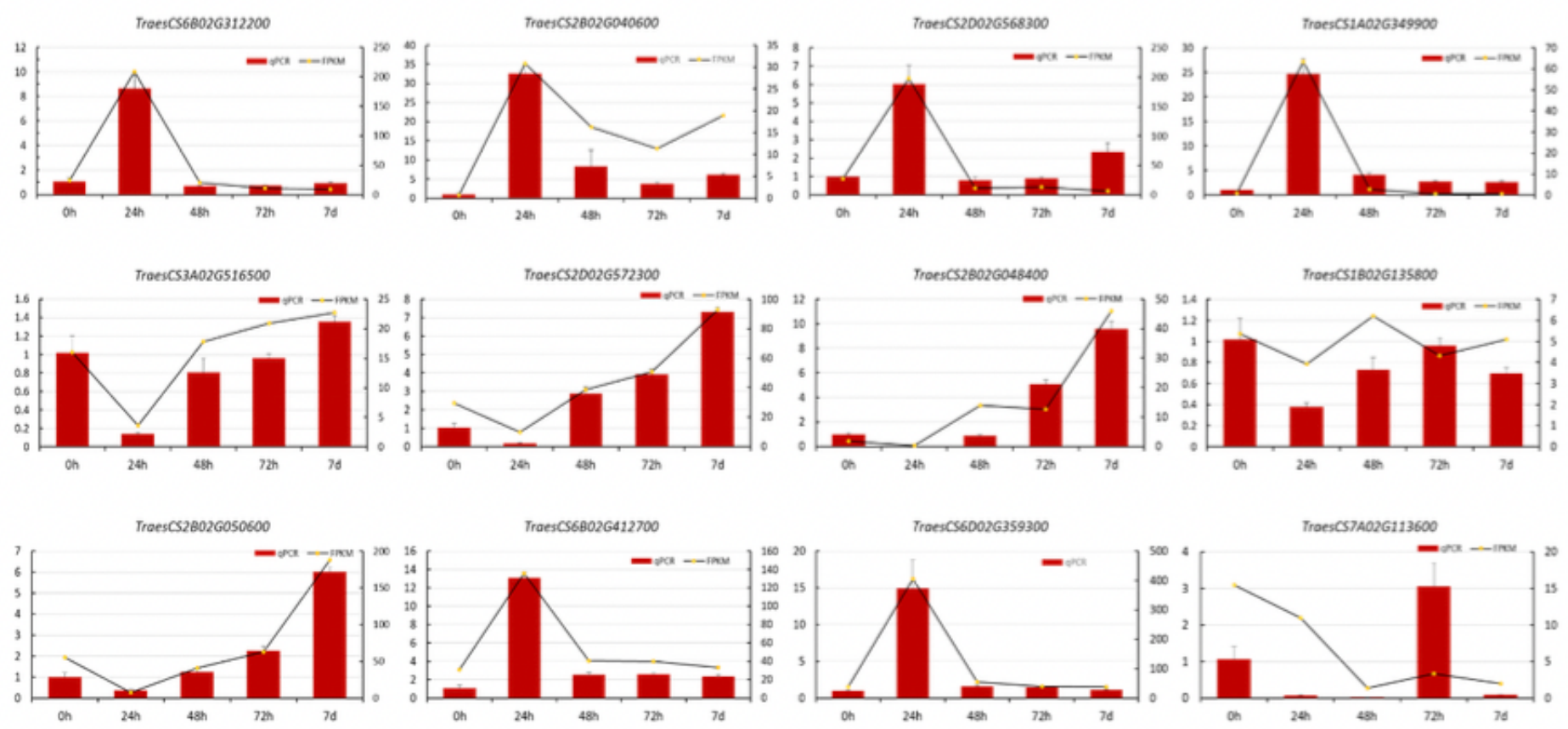

Figure 4

Expression patterns between qRT-PCR and RNA_seq for the 12 genes. The heights of the columns and points stand for the log2 (fold change) computed from both qRT-PCR and RNA_seq profile.
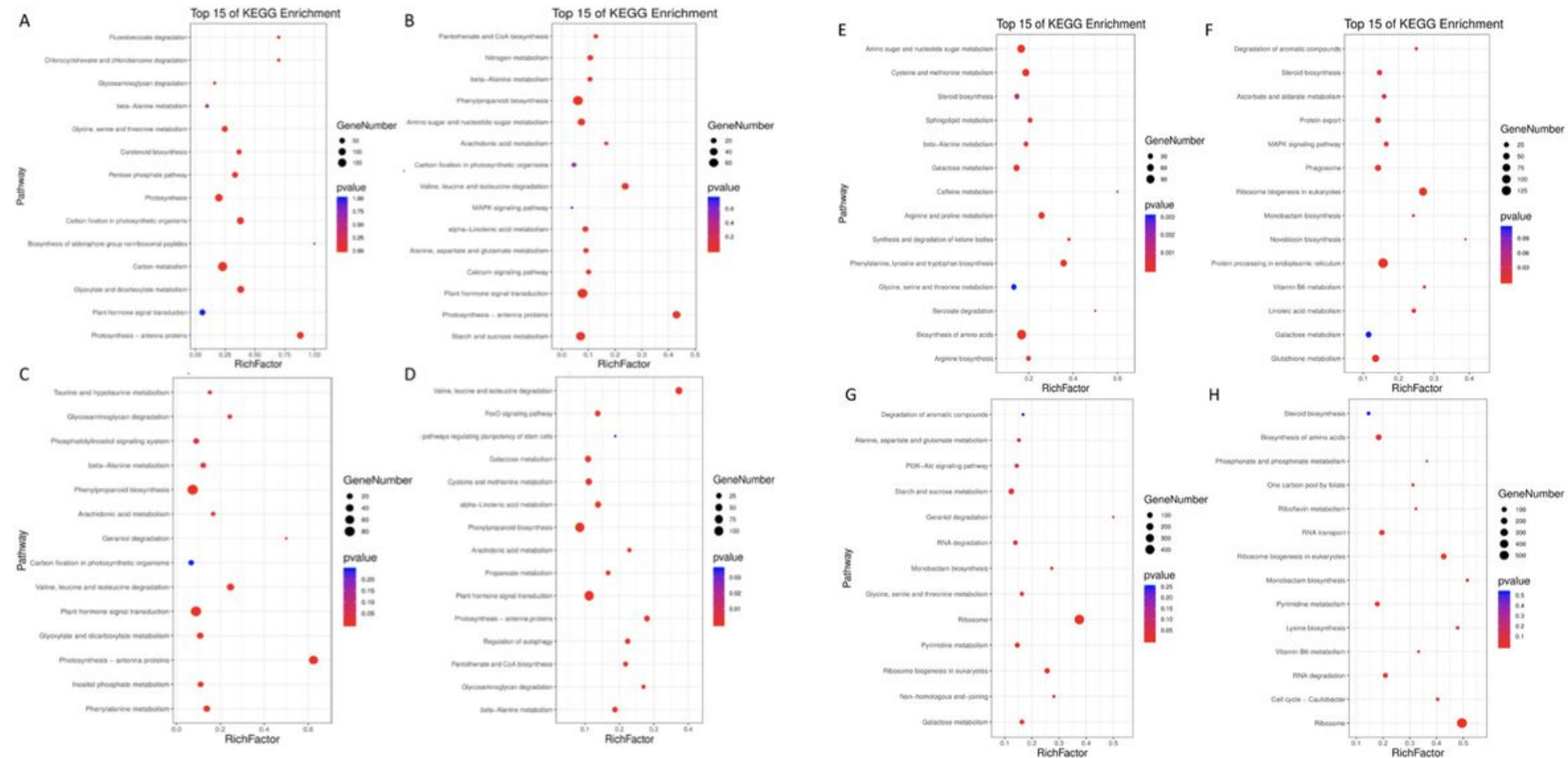

\section{Figure 5}

KEGG analysis of DEGs in CY12 at all time points. A-D are down-regulated DEGs enrichment in 24h (A), 48h (B), $72 \mathrm{~h}(\mathrm{C})$ and $7 \mathrm{~d}(\mathrm{D})$; E-H are up-regulated DEGs enrichment in 24h (E), 48h (F), 72h (G) and 7d (H). 

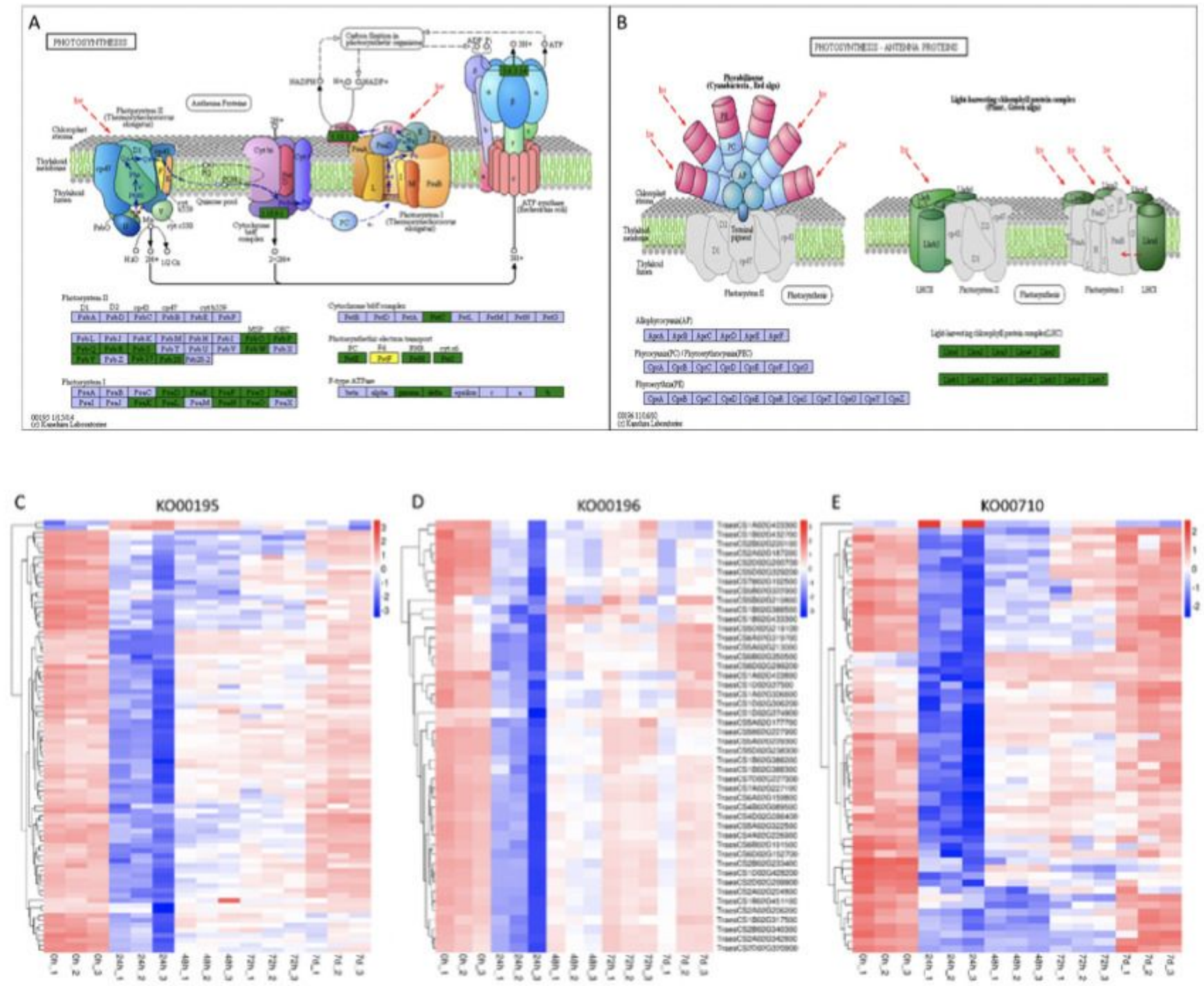

\section{Figure 6}

The DEGs enrichment in photosynthesis (A), photosynthesis-antenna proteins (B) and carbon fixation photosynthetic organisms; The heatmap of expression DEGs enrichment in K000195 (C), K000196 (D) and $\mathrm{KO00710} \mathrm{(E)} \mathrm{at} \mathrm{0h,} \mathrm{24h,} \mathrm{48h,} \mathrm{72h} \mathrm{and} \mathrm{7d} \mathrm{(B)} \mathrm{after} \mathrm{Pst-CYR34} \mathrm{inoculation,} \mathrm{the} \mathrm{expression} \mathrm{levels} \mathrm{estimated} \mathrm{using}$ log2(fold change) for each transcript. In figure A and figure B, genes marked with green was down-regulated in CY12. 

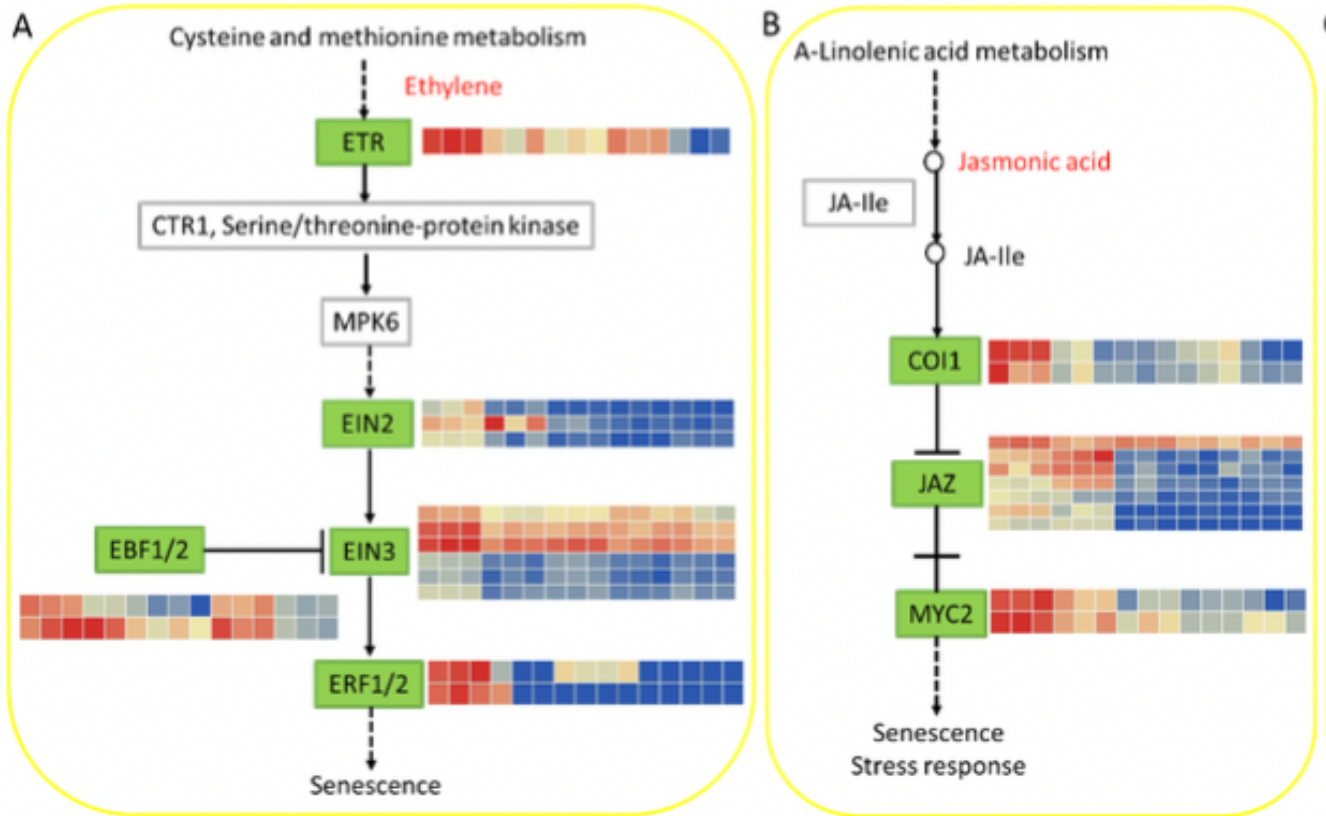

C

Phenylalanine metabolism

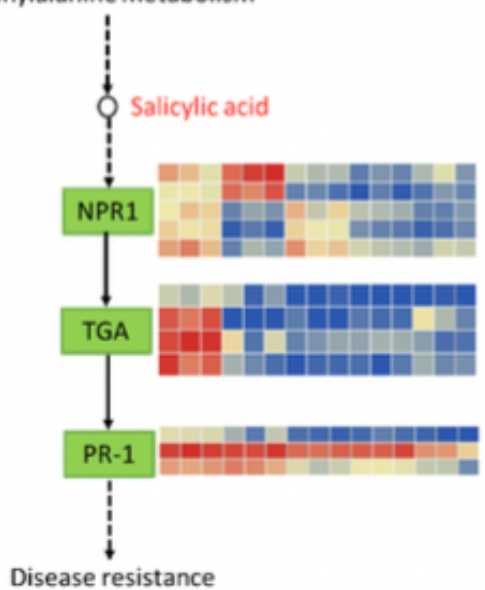

Figure 7

The DEGs expression enriched in ethylene (A), jasmonic acid (JA) (B) and salicylic acid (SA) (C) pathway, expression levels are indicated by the heatmap at $0 \mathrm{~h}, 24 \mathrm{~h}, 48 \mathrm{~h}, 72 \mathrm{~h}$ and $7 \mathrm{~d}$ group, estimated using log2(fold change) for each transcript. Genes marked with green was down-regulated in CY12.

A

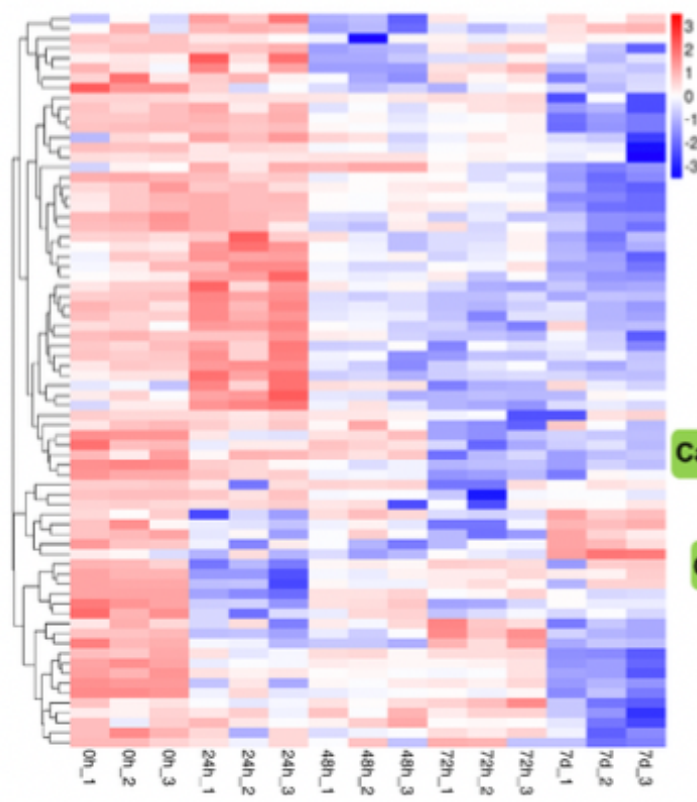

B

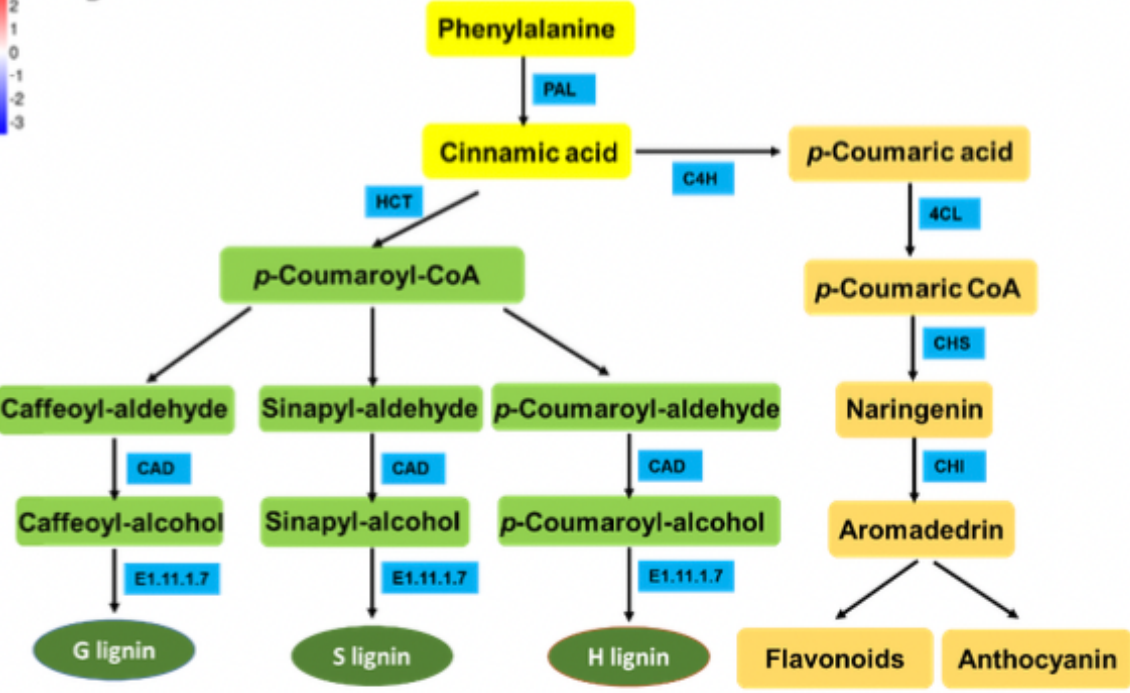

\section{Figure 8}

The heatmap of expression DEGs enrichment in phenylalanine pathway at $0 \mathrm{~h}, 24 \mathrm{~h}, 48 \mathrm{~h}, 72 \mathrm{~h}$ and $7 \mathrm{~d}$ in $\mathrm{CY} 12$ after Pst-CYR34 inoculation (A), and overview of specially DEGs enriched in phenylalanine pathway (B). 
This is a list of supplementary files associated with this preprint. Click to download.

- SupplementalFigures.docx

- Tables1.xlsx

- SupplementalFile3.xlsx

- SupplementalFile2.xIsx

- SupplementalFile1.xIsx

- SupplementalFile4.xIsx

- SupplementalFile5.xIsx 